\title{
ELLIPTICAL MULTIELECTRON DIMPLES ON LIQUID AND SOLID HELIUM SURFACES
}

\author{
V.B. Shikin \\ Insttitute of Solid State Physics, Academy of Sciences of the USSR, Chernogolovka, \\ Moscow district 142432, U.S.S.R. \\ and \\ P. Leiderer \\ Institut für Physik, Johannes Gutenberg-Universität, 6500 Mainz, West Germany
}

\begin{abstract}
The structure of multielectron dimples on helium substrates is investigated for conditions deviating from axial symmetry. Main sources for an ellipticity of dimples are an anisotropy of the surface tension, as for the liquid-solid He interface, or anisotropic boundary conditions, as for liquid He between vertical parallel plates.
\end{abstract}

WHEN ELECTRONS on liquid or solid helium are exposed to high electric fields normal to the helium surface, a transition is observed from a homogeneous distribution of the electrons to the so-called "dimple phase". In this state the electrons are localized, in clusters of up to $10^{7}$, in macroscopic dimples whose size is of the order of the capillary length [1] of the substrate, $0.5 \mathrm{~mm}$ for ${ }^{4} \mathrm{He}$ at $1 \mathrm{~K}$. The existing theory of such multielectron dimples $[2,3]$ has been formulated under the assumption of cylindrical symmetry of both the charge distribution and the surface deformation. Yet there are examples of dimples which require an extension to the more general case of elliptical geometry. We have in mind here the multielectron dimples on the solid-liquid interface (which deviate from circular shape due to the anisotropy of the surface tension of solid helium [4]), elliptical deformations of dimples when eigenfrequency oscillations are excited, or dimples in the presence of additional external one-dimensional perturbations etc. In this communication a theoretical treatment of two of these elliptical dimple systems and a qualitative experimental example are presented.

1. We first consider the structure of dimples at the ${ }^{4} \mathrm{He}$ solid-liquid interface. Taking into account earlier theoretical results of the dimple problem $[2,3]$ we can start from the following set of equations:

$e E_{\perp} \xi(x, y)+e^{2} \int \frac{n(x, y) d x^{\prime} d y^{\prime}}{\left[\left(x-x^{\prime}\right)^{2}+\left(y-y^{\prime}\right)^{2}\right]^{1 / 2}}=\lambda$,

$\alpha_{1} \frac{\partial^{2} \xi}{\partial x^{2}}+\alpha_{2} \frac{\partial^{2} \xi}{\partial y^{2}}+\Delta \rho g \xi=e E_{\perp} n(x, y)$,

$$
\begin{aligned}
& \left.\frac{\partial \xi}{\partial x}\right|_{0}=\left.\frac{\partial \xi}{\partial y}\right|_{0}=0 ; \quad \xi(x \rightarrow \infty)=\xi(y \rightarrow \infty)=0, \\
& \int n(x, y) \mathrm{d} x \mathrm{~d} y=N
\end{aligned}
$$

where we have assumed that the solid-liquid interface is characterized by two principal axes $x$ and $y$ perpendicular to each other, ${ }^{*}$ and $\alpha_{1}$ and $\alpha_{2}$ are the corresponding values of the interfacial tension. Here $\xi(x, y)$ is the deformation of the interface due to the localized charges, $n(x, y)$ is the local electron density in the dimple, $E_{\perp}$ is the electric pressing field normal to the interface, $\lambda$ is a Lagrange factor arising from the condition to fulfil equation (4), $N$ is the total number of charges in the dimple, $\Delta \rho$ is the density difference between solid and liquid herlium and $g$ is the acceleration due to gravity.

In the vicinity of the dimple minimum $(x=0$, $y=0$ ) an expansion of the deformation $\xi$ yields, in the harmonic approximation,

$$
\begin{aligned}
\xi(x, y) & =\xi(0)+\frac{1}{2} \xi_{x}^{\prime \prime}(0) x^{2}+\frac{1}{2} \xi_{y}^{\prime \prime}(0) y^{2}+\ldots \\
& =\xi(0)+A x^{2}+B y^{2}+\ldots .
\end{aligned}
$$

Equation (1) then reduces to an expression analogous to the basic equation of the Hertz contact problem in the theory of elasticity [5]. Using this analogy we immediately obtain from equations (1) and (5)

* The case treated here thus refers to a crystal orientation such that the interface has rectangular symmetry. 


$$
\begin{aligned}
& n(x, y)=\frac{3 N}{2 \pi a b}\left(1-\frac{x^{2}}{a^{2}}-\frac{y^{2}}{b^{2}}\right)^{1 / 2}, \\
& e E_{\perp} A=\frac{2 b}{\left(b^{2}-a^{2}\right) a^{2}} \frac{3 \pi}{4} N e^{2} E\left\{\left(1-\frac{a^{2}}{b^{2}}\right)^{1 / 2}\right\}, \\
& e E_{\perp} B=\frac{2}{\left(b^{2}-a^{2}\right) b} \frac{3 \pi}{4} N e^{2} K\left\{\left(1-\frac{a^{2}}{b^{2}}\right)^{1 / 2}\right\},
\end{aligned}
$$

where $a$ and $b$ are the main axes of the elliptical charge distribution $(a<b)$, and $E\left\{\left(1-a^{2} / b^{2}\right)^{1 / 2}\right\}$ and $K\left\{\left(1-a^{2} / b^{2}\right)^{1 / 2}\right\}$ are elliptic integrals.

In order to determine the values of the 4 parameters $a, b, A$ and $B$ one needs, in addition to equations (7) and (8), two additional relations, which can be derived using equations (2) and (5)

$2 \alpha_{1} A+2 \alpha_{2} B \simeq e E_{\perp} n(0)=e E_{\perp} \frac{3 N}{2 \pi a b}$,

$\alpha_{1} b^{2}=\alpha_{2} a^{2}$.

Equation (10) follows from the symmetry of the problem, whereas in equation (9) the gravitational term has been neglected and use has been made of the fact that the charge is concentrated in the centre of the dimple, with the largest diameter of the charge distribution, $b$, being distinctly smaller than the capillary length $\kappa^{-1}=$ $(\alpha / \Delta \rho g)^{1 / 2 *}$.

The problem of elliptical dimples on an anisotropic substrate, formulated in terms of equations (1) and (2), thus reduces to the solution of the set (7)-(10).

The above relations obviously include the isotropic case of liquid helium substrates. Setting $\alpha_{1}=\alpha_{2}=\alpha$, we obtain from equation (10)

$a=b=: R$,

equations (7) and (8) lead to

$\xi^{\prime \prime}(0)=\frac{3 \pi e N}{4 R^{3} E_{\perp}}$

and equation (9) reduces to

$2 \alpha \xi^{\prime \prime}(0)=e E_{\perp} n(0)=e E_{\perp} \frac{3 N}{2 \pi R^{2}}$.

The radius $R$ and density $n(r)$ of the charge distribution in an isotropic dimple therefore is given by (cf. also

$[2,3])$

$R=\frac{\pi^{2} \alpha}{E_{\perp}^{2}}$,

* In the dimple centre the electrostatic pressure due to the charges [ $\mathrm{r}$.h.s. of equation (9)] is mainly balanced by the pressure resulting from the curvature of the interface. The gravitational term $\Delta \rho g \xi$ from equation (2) only gives rise to a relative contribution of the order of $\kappa^{2} b^{2} \ll 1$ (see also [2]). and

$n(r)=\frac{3 N}{2 \pi R^{2}}\left(1-\frac{r^{2}}{R^{2}}\right)^{1 / 2}$.

If the anisotropy of the substrate surface tensionis not too large - as probably for solid helium [4] - it can be written as

$\alpha_{1}=\alpha(1-\Delta)$,

$\alpha_{2}=\alpha(1+\Delta)$,

with $\Delta \ll 1$. Then the solution of equations (7)-(10) gives for the main axes of the elliptical charge distribution

$a=R\left(1-\frac{\Delta}{2}\right)$,

$b=R\left(1+\frac{\Delta}{2}\right)$.

An experimental study of the anisotropy of dimples on solid helium therefore should allow to determine the anisotropic surface tension of this quantum crystal.

2. As a second example for the formation of elliptical multielectron dimples we consider a charged helium surface subject to anisotropic boundary conditions (see Figs. 1 and 2), as, e.g., a charge helium surface between two parallel vertical plates at a distance $d$. For small plate distances $\left(d \ll \kappa^{-1}\right)$ the curvature of the helium surface in the $x$-direction, perpendicular to the plates, is [1]

$A=\frac{1}{2} \xi_{x}^{\prime \prime}(0) \simeq \frac{1}{d}$.

a)

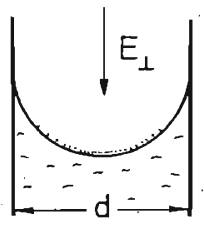

b)

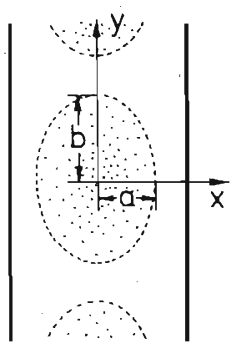

Fig. 1. Schematic side (a) and top (b) view of a charged helium surface between two vertical walls, subject to a vertical electric field $E_{1}$. The dashed line in (b) represents the boundary of the elliptic electron distribution, which also leads to elliptical contour lines of the dimple profile. 


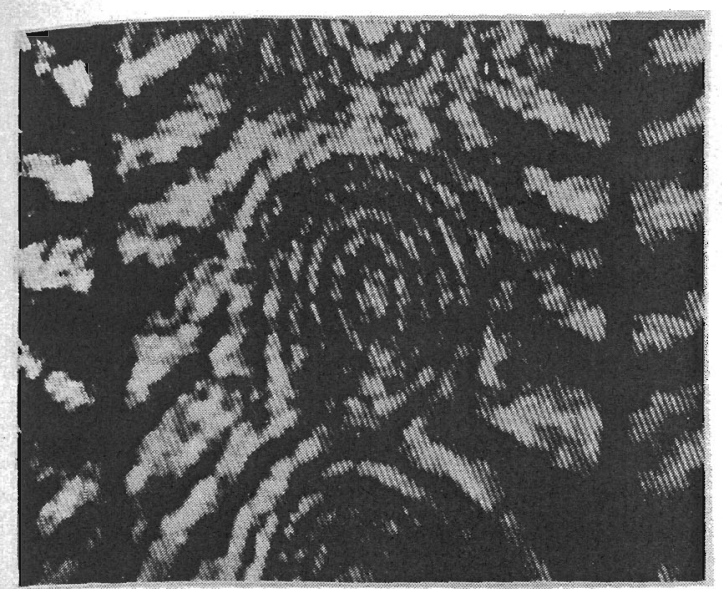

Fig. 2. Photograph of elliptical multielectron dimples on liquid ${ }^{4} \mathrm{He}$ at a temperature $T=3 \mathrm{~K}$. Instead of the mechanical side walls considered in Fig. 1 here two charged wires (appearing as vertical straight lines, distance $d=2.7 \mathrm{~mm}$ ) provide the potential for confining the electrons from the side, thus introducing the anisotropy of the dimples. The interference fringes, which represent contour lines of the dimple profile, were obtained as described in [6]. Consecutive fringes correspond to a height difference of $11 \mu \mathrm{m}$.

When the electric field is high enough that dimples are formed, a finite curvature of the surface also appears in the direction parallel to the plates

$B=\frac{1}{2} \xi_{y}^{\prime \prime}(0) \simeq e E_{\perp} \frac{3 N}{4 \pi a b \alpha}$,

where again $a$ and $b$ are the axes of the elliptical electron distribution in the dimple.

Using the relations for $A(14)$ and $B(15)$ and the general conditions (7) and (8), we have in the limiting case $b \gg a$

$$
b=\frac{3 \pi^{2} d e N}{4 a^{2} E_{\perp}},
$$

and $a^{5}=\frac{9 \pi d^{2} e^{2} N^{2}}{16 \alpha} \ln \frac{b}{a}$.

If, for example, a dimple containing $N \simeq 10^{3}$ electrons in an applied electric field $E \simeq 3000 \mathrm{~V} \mathrm{~cm}^{-1}$ is located between two plates at a distance $d \simeq 10^{-4} \mathrm{~cm}$, then the electron distribution is characterized by $a \lesssim 10^{-4} \mathrm{~cm}$ and $b \simeq 10^{-3} \mathrm{~cm}$, resulting in an anisotropy $v / a \simeq 10$.

In addition to the elliptical shape of multielectron dimples between parallel plates also other essential properties of these macroscopic quasi-one-dimensional systems will deviate from the isotropic helium surfaces treated earlier $[2,3]$. In particular, it should be possible in restricted geometries (electrons between parallel plates or also electrons in a system of vertical cylindrical capillaries) to suppress the gravitational instability of the charged liquid surface and thus reach considerably higher electron densities than accessible until now. The investigation of these problems will be presented in a separate paper.

Acknowledgements - We appreciate the assistance of $\mathrm{J}$. Bodensohn and S. Herminghaus, and financial support by the Deutsche Forschungsgemeinschaft. One of us (VBS) is especially grateful to Professor Koch for the kind invitation to his institute at the Technical University of Munich where a major part of this work was completed.

\section{REFERENCES}

1. L.D. Landau \& E.M. Lifshitz, Fluid Mecrianics, pp. 235. Pergamon (1959).

2. V.B. Shikin \& P. Leiderer, Pis'ma ZhETF 32, 439 (1980); [JETP Lett. 32, 416 (1980)]; V.B. Shikin \& P. Leiderer, ZhETF 81, 184 (1981); [Sov. Phys. -JETP 54, 92 (1981)].

3. V.L. Mel'nikov \& S.V. Meshkov, ZhETF 81, 951 (1981); [Sov. Phys. - JETP 54, 505 (1981)].

4. D. Savignac \& P. Leiderer, Phys. Rev. Lett. 49, 1869 (1982).

5. L.D. Landau \& E.M. Lifshitz, Theory of Elasticity, p. 30. Pergamon (1959).

6. P. Leiderer, W. Ebner \& V.B. Shikin, Surf. Sci. 113, 405 (1982). 